\title{
48. ABUNDANCE PROFILES OF IRIDIUM AND OTHER ELEMENTS NEAR THE CRETACEOUS/TERTIARY BOUNDARY IN HOLE S16F OF DEEP SEA DRILLING PROJECT LEG 72 ${ }^{1}$
}

\author{
H. V. Michel and F. Asaro, Lawrence Berkeley Laboratory, University of California, Berkeley, California \\ W. Alvarez, Department of Geology and Geophysics, University of California, Berkeley, California \\ L. W. Alvarez, Space Sciences Laboratory and Lawrence Berkeley Laboratory, University of California, \\ Berkeley, California \\ and \\ D. A. Johnson, Woods Hole Oceanographic Institution, Woods Hole, Massachusetts
}

\section{INTRODUCTION}

Anomalously high values of Ir have been detected in association with the Cretaceous/Tertiary boundary in about a dozen marine or continental sections laid down about $65 \mathrm{Ma}$ in many different parts of the world (Alvarez et al., 1981). One possible exception is the Cretaceous/Tertiary boundary region in DSDP Site 356 , in the South Atlantic off the coast of South America. In a detailed study of that section, the maximum Ir abundance was about $0.2 \mathrm{ppb}$, much lower than the $3-90 \mathrm{ppb}$ found in most of the other marine locations (authors' unpublished data on the Cretaceous/Tertiary region of Site 356,1982 ). A study of Hole $516 \mathrm{~F}$, which is not too far from Site 356, could show whether the very low (or missing) Cretaceous/Tertiary Ir anomaly at Site $\mathbf{3 5 6}$ is characteristic of this region of the South Atlantic.

\section{CORE LITHOLOGY AND STRATIGRAPHY}

The position of the Cretaceous/Tertiary boundary at Hole $516 \mathrm{~F}$ may be determined quite precisely, since calcareous nannoplankton and planktonic foraminifers are moderately to well preserved throughout virtually the entire pelagic sequence on the Rio Grande Rise. The boundary occurs in Section 516F-89-5 (Fig. 1), and the microfossil assemblages give no evidence of any hiatus between the Maestrichtian and the Danian (Paleocene).

In the split cores, there were no detectable artifacts of reworking that may have been introduced by bioturbation in situ or by drilling brecciation. In addition, the microfossil assemblages show no co-occurrences of Cretaceous and Paleocene marker species in samples near the boundary. Thus, we are confident that the "boundary" recovered in the cores from Site 516 is reasonably representative of in situ deposition conditions, with minimal post-depositional vertical reworking or mixing of lithologies.

Biostratigraphically the Cretaceous/Tertiary boundary may be identified as follows: nannofossil assemblages show that the oldest zone of the Paleocene (Markalius inversus Zone, NP1) extends down to Sam-

\footnotetext{
${ }^{1}$ Barker, P. F., Carlson, R. L., Johnson, D. A., et al., Init. Repts. DSDP, 72; Washington (U.S. Govt. Printing Office).
}

ple $516 \mathrm{~F}-89-5,33.5 \mathrm{~cm}$; the youngest Cretaceous zone (Micula mura zone) begins at Sample 516F-89-5, 34.5 $\mathrm{cm}$ and continues downward. Planktonic foraminifers are relatively rare in the basal Paleocene. Several small specimens $(<150 \mu)$ described as Eoglobigerina sp. are present in Samples 516F-89-5, 21-24 cm and 24-26 cm (Pujol, this volume); this is consistent with a basal Cenozoic age, Zone Pla. The characteristic Cretaceous planktonic foraminifers (e.g., Globotruncana spp.) are present in Sample $516 \mathrm{~F}-89-5,28-30 \mathrm{~cm}$, and extend downward below this level. More extensive documentation of the calcareous microfloral and microfaunal successions around the Cretaceous/Tertiary boundary in Hole $516 \mathrm{~F}$ is presented in separate chapters in this volume (Weiss, Pujol).

The lithologic sequence in the vicinity of the Cretaceous/Tertiary boundary may be summarized as follows: the lower Paleocene sediment is generally a reddish brown (5 YR 5/3) partially dolomitized microcrystalline limestone. The interval directly above the boundary in Sample 516F-89-5, 27-33 cm (Fig. 1) is cut by a complex sequence of gray and reddish brown oblique laminations, denoting a transition zone to the underlying light gray limestone. At Sample 516F-89-5, $34.0-34.5 \mathrm{~cm}$, there is a thin dark gray (5 YR 5/1), clayrich layer with sharp and irregular upper and lower contacts. The uppermost Maestrichtian limestone is light gray to white, showing common bioturbation structures in some intervals, and lacking the secondary intrastratal solution structures that are common above the Cretaceous/Tertiary boundary. It is noteworthy that, at Site 516 , there is no single distinctive "boundary clay"; instead, there are a number of darker-colored layers and laminations in Sample 516F-89-5, 26-35 cm (Fig. 1), which are presumably more clay rich than the surrounding limestone. The thin, dark gray clay at Sample 516F89-5, 34.0-34.5 cm, however, does appear to correspond with the Cretaceous/Tertiary boundary according to nannofossil biostratigraphy (Fig. 1).

\section{METHODS}

\footnotetext{
Sampling

Sixteen samples from Interval $516 \mathrm{~F}-89-5,11-130 \mathrm{~cm}$, and four other samples (Samples 516F-89-3, 64-66 cm; 516F-89-3, 129-130 cm;
} 


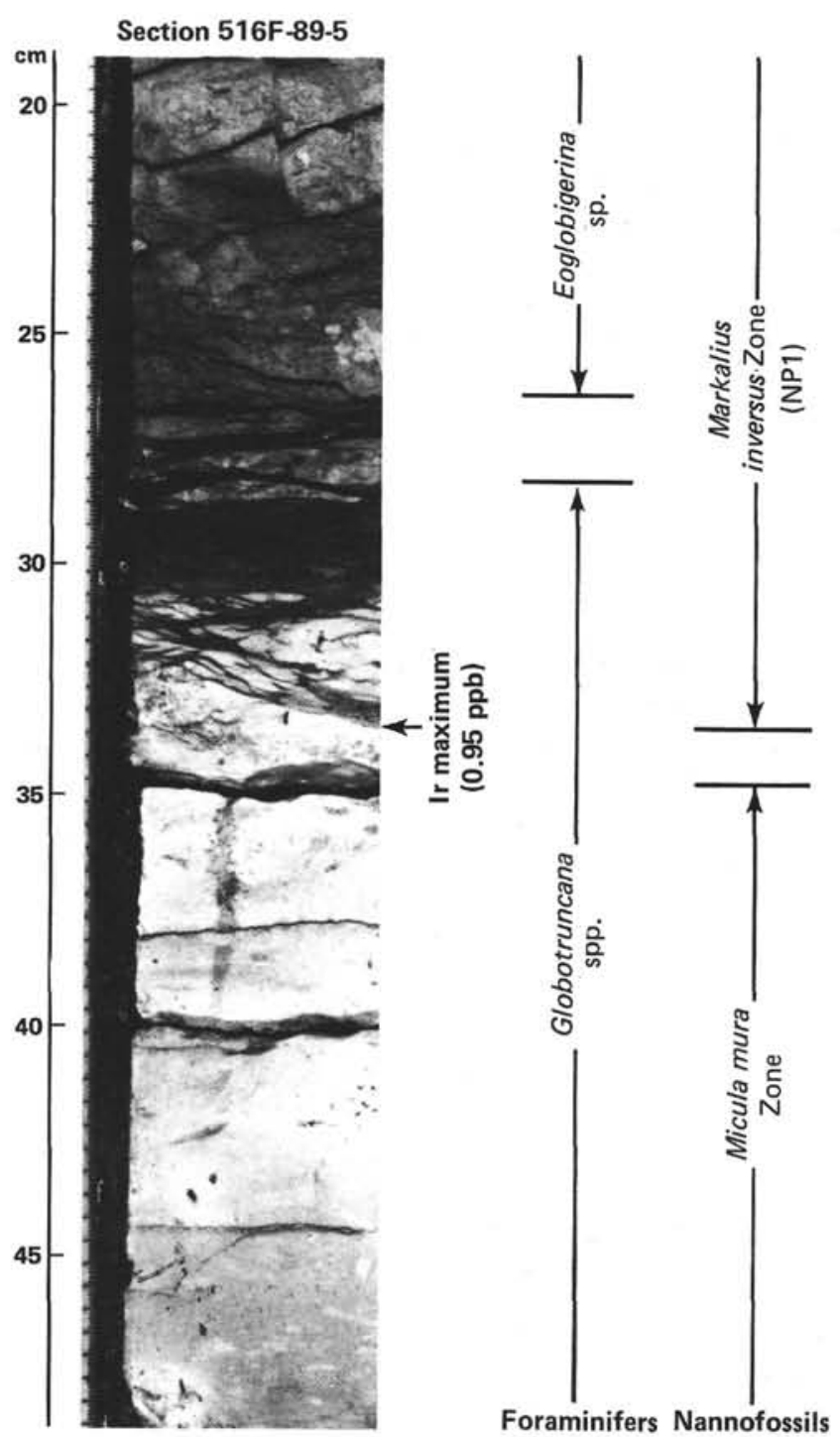

Figure 1. Photograph of Cretaceous/Tertiary boundary in Section 516F-89-5. Ranges of diagnostic foraminifers and nannofossils are shown, based on reports by Pujol and Weiss (this volume).

$516 \mathrm{~F}-89-4,78.5-80 \mathrm{~cm}$; and $516 \mathrm{~F}-89-4,90-92 \mathrm{~cm}$ ) were dried at about $130^{\circ} \mathrm{C}$ and ground with an agate mortar and pestle. Duplicate samples were taken from each of the ground powders. One sample of a duplicate set was used in a 20-minute low-flux neutron irradiation, and the duplicate was used in an 8-hour full-power neutron irradiation. These samples, which are indicated in Table 1 by the neutron activation analysis (NAA) pill name 2017 , were subsequently found to be contaminated with Ir from the platinum rings worn by the technician who ground and prepared them for NAA (Alvarez et al., 1982). New samples from the identical intervals were dried, ground, and prepared for NAA by a technician who did not wear rings, and they can be identified in Table 1 by the NAA pill names 2026, 2029, and 2032. Ir and a few other elements were measured in this resampling. A more extensive study of the region above and below the Cretaceous/Tertiary boundary in Hole 516F, Cores 83-89 and 90-94, was made. These samples were dried and ground as the others and are shown in Table 1 with the NAA pill name 2031. When high-precision techniques of NAA are used and fractionation of different density phases can be avoided, duplicate samples taken from a ground powder will usually have abundance values nearly consistent with the statistical uncertainties in the counting of gamma rays. Although fractionation effects can be particularly severe in ball mills that utilize centrifugal forces
Table 1. Concordance of sample and NAA pill names, Leg 72 , Hole $516 \mathrm{~F}$

\begin{tabular}{|c|c|c|c|}
\hline $\begin{array}{l}\text { DSDP sample } \\
\text { core-section } \\
\text { (interval in } \mathrm{cm} \text { ) }\end{array}$ & NAA sample & NAA pill & \\
\hline $83-5,69-70$ & DSEA-103 & 2031-E & \\
\hline $84-5,69-70$ & -104 & $-F$ & \\
\hline $85-2,69-70$ & -105 & $-G$ & \\
\hline $86-5,69-70$ & -106 & $-\mathrm{H}$ & \\
\hline $87-5,69-70$ & -107 & $-\mathrm{J}$ & \\
\hline $89-3,64-66$ clay & -22 & 2032-E & 2017-E \\
\hline $89-3,128-130$ & -23 & $-F$ & $-F$ \\
\hline $89-4,78.5-80$ & -24 & $-G$ & $-\mathrm{G}$ \\
\hline 90-92 clay & -25 & $-\mathrm{H}$ & $-\mathrm{H}$ \\
\hline $89-5,11-12.5$ & -26 & 2029-Q & $-\mathrm{J}$ \\
\hline $16-17$ & -27 & $-\mathbf{R}$ & $-\mathrm{K}$ \\
\hline $18-20$ & -28 & $-S$ & $-\mathrm{M}$ \\
\hline $20-22$ & -29 & $-\mathrm{T}$ & $-\mathrm{N}$ \\
\hline $22.5-24.5$ & -30 & 2026-EFGH & $-\mathrm{O}$ \\
\hline $24.5-26$ & -31 & 2029-U & $-\mathrm{P}$ \\
\hline $27-28$ & -32 & 2032-J & $-Q$ \\
\hline $29-30$ & -33 & $-\mathrm{K}$ & $-\mathrm{R}$ \\
\hline $30-32$ & -34 & 2026-JKMN & $-\mathrm{S}$ \\
\hline $32-32.5$ & -113 & 2031-Q & \\
\hline $32.5-33.0$ & -114 & $-\mathbf{R}$ & \\
\hline $33.0-33.5$ & -115 & $-S$ & \\
\hline $33.5-34.0$ & -116 & $-\mathrm{T}$ & \\
\hline $34-35.5$ & -35 & 2032-M & $2017-\mathrm{T}$ \\
\hline $34.5-36.5$ & -36 & $-\mathrm{N}$ & $-\mathrm{U}$ \\
\hline $38-39$ & -37 & -0 & $-\mathrm{V}$ \\
\hline $39.5-40.5$ & 38 & $-P$ & $-\mathrm{W}$ \\
\hline $45-46$ & -39 & $-Q$ & $-\mathrm{X}$ \\
\hline $49.5-50.5$ & -40 & $-\mathrm{R}$ & $-\mathrm{Y}$ \\
\hline $129-130$ & -41 & 2026-OPQR & $-\mathrm{Z}$ \\
\hline $90-5,69-70$ & -108 & 2031-K & \\
\hline $91-5,69-70$ & -109 & $-M$ & \\
\hline $92-4,69-70$ & -110 & $-\mathrm{N}$ & \\
\hline $93-5,69-70$ & -111 & -0 & \\
\hline $94-5,69-70$ & -112 & $-P$ & \\
\hline
\end{tabular}

Note: $\mathrm{NAA}=$ neutron activation analysis.

perpendicular to the gravitational field for the grinding action, hand grinding with a mortar and pestle has a much smaller effect.

\section{Measurements}

All samples were measured by high-precision methods of neutron activation analysis (Perlman and Asaro, 1969; Perlman and Asaro, 1971; Yellin et al., 1978; Asaro et al., 1981). Most elements were calibrated against a multielement standard, "STANDARD POTTERY" (Perlman and Asaro, 1971). Chlorine and calcium were calibrated against primary standards, and Ir was calibated against a secondary Ir standard, DINO-1, made from the Cretaceous/Tertiary boundary layer at Stevns Klint (Alvarez et al., 1982). The data, except for some on the rare earths that are still being processed, are shown in Tables 2 and 3. Seawater contributions for $\mathrm{Na}$ were deduced from the $\mathrm{Cl}$ abundances and subtracted from the Na values. The listed errors, which are estimates of the standard deviations, correspond to either the statistical uncertainties in counting gamma rays or $1 \%$ of the abundances, whichever are larger. The precisions of measurement are about equal to these errors. The accuracies are usually close to the precisions for elements calibrated against primary or secondary standards. Zn, Se, and $\mathrm{Mg}$ were calibrated against flux monitors and contain about $10 \%$ additional uncertainties.

\section{Sampling Homogeneity}

In one set of the 2017 samples run, $\mathrm{Ba}$ abundances were measured (after a low-flux irradiation) with the gamma rays of ${ }^{139} \mathrm{Ba}$ and, in the duplicate set, (after a high-flux irradiation) with the gamma rays of ${ }^{131} \mathrm{Ba}$. In 16 comparisons, 8 were within 1 standard deviation (the statistical error in counting gamma rays), 7 disagreed by between 1 
Table 2. Major element abundances expressed in percent.

\begin{tabular}{|c|c|c|c|c|c|c|c|c|}
\hline $\begin{array}{l}\text { Sample } \\
\text { number }\end{array}$ & $\mathrm{Ca}$ & Al & $\mathrm{Cl}$ & $\mathrm{Mg}$ & $\mathrm{Na}$ & K & $\mathrm{Ti}$ & $\mathrm{Fe}$ \\
\hline SEA-103 & $31.4 \pm 0.8$ & $1.482 \pm 0.033$ & $0.262 \pm 0.023$ & $<1.2$ & $0.289 \pm 0.016$ & $0.66 \pm 0.08$ & $0.090 \pm 0.022$ & $0.685 \pm 0.010$ \\
\hline-104 & $32.5 \pm 0.7$ & $1.445 \pm 0.034$ & $0.270 \pm 0.017$ & $0.59 \pm 0.24$ & $0.273 \pm 0.013$ & $0.52 \pm 0.08$ & $0.074 \pm 0.021$ & $0.765 \pm 0.011$ \\
\hline-105 & $35.6 \pm 0.9$ & $0.82 \pm 0.06$ & $0.375 \pm 0.024$ & $<1.2$ & $0.172 \pm 0.015$ & $0.31 \pm 0.08$ & $0.059 \pm 0.035$ & $0.477 \pm 0.008$ \\
\hline-106 & $36.4 \pm 1.0$ & $0.65 \pm 0.08$ & $0.387 \pm 0.023$ & $0.98 \pm 0.34$ & $0.134 \pm 0.015$ & $0.23 \pm 0.07$ & $<0.12$ & $0.569 \pm 0.009$ \\
\hline-107 & $33.9 \pm 1.0$ & $1.23 \pm 0.11$ & $0.241 \pm 0.022$ & $1.00 \pm 0.38$ & $0.195 \pm 0.014$ & $0.68 \pm 0.07$ & $<0.09$ & $1.044 \pm 0.013$ \\
\hline-22 & $9.0 \pm 0.5$ & $7.82 \pm 0.09$ & $0.239 \pm 0.030$ & $1.6 \pm 0.6$ & $0.874 \pm 0.026$ & $2.68 \pm 0.22$ & $0.52 \pm 0.04$ & $4.09 \pm 0.05$ \\
\hline-23 & $12.4 \pm 1.2$ & $9.96 \pm 0.17$ & - & - & - & - & - & $2.538 \pm 0.034$ \\
\hline-24 & $4.54 \pm 0.38$ & $7.82 \pm 0.11$ & $0.238 \pm 0.021$ & $2.2 \pm 0.5$ & $0.796 \pm 0.022$ & $3.09 \pm 0.18$ & $0.495 \pm 0.038$ & $4.45 \pm 0.06$ \\
\hline-25 & $7.3 \pm 0.4$ & $7.42 \pm 0.12$ & $0.223 \pm 0.020$ & $<1.8$ & $0.739 \pm 0.020$ & $3.20 \pm 0.18$ & $0.501 \pm 0.039$ & $3.86 \pm 0.05$ \\
\hline-26 & $34.4 \pm 0.9$ & $1.15 \pm 0.08$ & $0.178 \pm 0.020$ & $<1.0$ & $0.114 \pm 0.012$ & $0.38 \pm 0.16$ & $0.095 \pm 0.040$ & $0.84 \pm 0.06$ \\
\hline-27 & $35.1 \pm 0.8$ & $1.095 \pm 0.025$ & $0.193 \pm 0.035$ & $<0.7$ & $0.121 \pm 0.020$ & $0.34 \pm 0.16$ & $0.084 \pm 0.021$ & $0.746 \pm 0.012$ \\
\hline-28 & $32.8 \pm 0.8$ & $1.87 \pm 0.04$ & $0.171 \pm 0.034$ & $0.72 \pm 0.32$ & $0.210 \pm 0.020$ & $0.69 \pm 0.16$ & $0.124 \pm 0.025$ & $1.19 \pm 0.08$ \\
\hline-29 & $30.6 \pm 0.8$ & $1.87 \pm 0.05$ & $0.181 \pm 0.032$ & $<1.3$ & $0.215 \pm 0.019$ & $0.91 \pm 0.16$ & $0.146 \pm 0.028$ & $1.30 \pm 0.08$ \\
\hline-30 & $35.1 \pm 0.9$ & $1.40 \pm 0.06$ & $0.162 \pm 0.031$ & $<1.1$ & $0.153 \pm 0.018$ & $0.50 \pm 0.15$ & $0.115 \pm 0.031$ & $0.88 \pm 0.07$ \\
\hline-31 & - & - & $0.100 \pm 0.031$ & - & $0.216 \pm 0.018$ & $0.97 \pm 0.15$ & $0.089 \pm 0.034$ & $1.098 \pm 0.018$ \\
\hline-32 & $31.5 \pm 0.8$ & $1.215 \pm 0.033$ & $0.148 \pm 0.032$ & $<0.9$ & $0.174 \pm 0.019$ & $0.53 \pm 0.15$ & $0.122 \pm 0.024$ & $0.812 \pm 0.013$ \\
\hline-33 & $19.0 \pm 0.6$ & $5.01 \pm 0.08$ & $0.149 \pm 0.030$ & $0.95 \pm 0.36$ & $0.533 \pm 0.020$ & $2.40 \pm 0.17$ & $0.282 \pm 0.028$ & $2.278 \pm 0.031$ \\
\hline-34 & $27.5 \pm 0.9$ & $3.82 \pm 0.08$ & - & - & - & - & - & $1.331 \pm 0.020$ \\
\hline-113 & $27.6 \pm 0.7$ & $2.571 \pm 0.039$ & $0.256 \pm 0.022$ & $0.77 \pm 0.35$ & $0.282 \pm 0.015$ & $1.26 \pm 0.15$ & $0.135 \pm 0.022$ & $1.120 \pm 0.014$ \\
\hline-114 & $28.7 \pm 0.7$ & $2.38 \pm 0.05$ & $0.228 \pm 0.022$ & $1.33 \pm 0.33$ & $0.284 \pm 0.015$ & $1.06 \pm 0.15$ & $0.187 \pm 0.024$ & $0.976 \pm 0.013$ \\
\hline-115 & $14.6 \pm 0.5$ & $5.57 \pm 0.09$ & $0.178 \pm 0.020$ & $1.7 \pm 0.4$ & $0.654 \pm 0.019$ & $2.65 \pm 0.15$ & $0.658 \pm 0.034$ & $2.692 \pm 0.027$ \\
\hline-116 & $31.9 \pm 0.8$ & $1.93 \pm 0.07$ & $0.284 \pm 0.021$ & $<1.2$ & $0.240 \pm 0.014$ & $1.13 \pm 0.14$ & $0.123 \pm 0.029$ & $0.763 \pm 0.011$ \\
\hline-35 & $13.5 \pm 0.7$ & $6.12 \pm 0.12$ & - & - & - & - & - & $2.288 \pm 0.031$ \\
\hline-36 & $30.3 \pm 0.9$ & $1.68 \pm 0.11$ & $0.336 \pm 0.032$ & $0.90 \pm 0.3$ & $0.182 \pm 0.019$ & $0.86 \pm 0.15$ & $0.096 \pm 0$ & $0.766 \pm 0.013$ \\
\hline-37 & $30.5 \pm 0.7$ & $1.988 \pm 0.036$ & $0.259 \pm 0.027$ & $<0.9$ & $0.274 \pm 0.017$ & $1.20 \pm 0.18$ & $0.139 \pm 0.021$ & $0.999 \pm 0.016$ \\
\hline-38 & $32.1 \pm 0.9$ & $1.61 \pm 0.06$ & $0.346 \pm 0.034$ & $<1.3$ & $0.209 \pm 0.020$ & $0.71 \pm 0.18$ & $0.103 \pm 0.032$ & $0.855 \pm 0.014$ \\
\hline-39 & $31.4 \pm 0.9$ & $1.65 \pm 0.07$ & $0.282 \pm 0.032$ & $<0.9$ & $0.212 \pm 0.019$ & $0.78 \pm 0.17$ & $0.102 \pm 0.035$ & $1.033 \pm 0.016$ \\
\hline-40 & $31.1 \pm 1.0$ & $1.73 \pm 0.09$ & $0.326 \pm 0.034$ & $<1.5$ & $0.234 \pm 0.021$ & $0.76 \pm 0.18$ & $0.109 \pm 0.042$ & $1.220 \pm 0.018$ \\
\hline-41 & $26.4 \pm 1.1$ & $3.43 \pm 0.13$ & - & - & - & - & - & $1.682 \pm 0.024$ \\
\hline-108 & $30.1 \pm 0.7$ & $2.498 \pm 0.038$ & $0.203 \pm 0.024$ & $<1.1$ & $0.298 \pm 0.016$ & $1.17 \pm 0.08$ & $0.203 \pm 0.024$ & $1.474 \pm 0.017$ \\
\hline-109 & $29.2 \pm 0.7$ & $2.37 \pm 0.05$ & $0.201 \pm 0.023$ & $<1.2$ & $0.301 \pm 0.018$ & $1.23 \pm 0.08$ & $0.181 \pm 0.026$ & $1.486 \pm 0.017$ \\
\hline-110 & $24.7 \pm 0.7$ & $3.37 \pm 0.07$ & $0.238 \pm 0.022$ & $1.14 \pm 0.39$ & $0.367 \pm 0$ & $1.70 \pm 0$ & $0.222 \pm 0.030$ & $2.174 \pm 0.023$ \\
\hline-111 & $13.9 \pm 0.6$ & $5.52 \pm 0.10$ & $0.182 \pm 0.022$ & $<0.9$ & $0.619 \pm 0.019$ & $2.61 \pm 0.10$ & $0.301 \pm 0.036$ & $2.945 \pm 0.029$ \\
\hline-112 & $26.5 \pm 0.8$ & $2.58 \pm 0.09$ & $0.165 \pm 0.021$ & $0.9 \pm 0.4$ & $0.336 \pm 0.015$ & $1.37 \pm 0.08$ & $0.208 \pm 0.040$ & $1.984 \pm 0.021$ \\
\hline
\end{tabular}

Note: See Table 1 for corresponding DSDP sample numbers. A flux monitor was used for calibration of Mg. A seawater contribution of 0.556 times the $\mathrm{Cl}$ abundance has been removed from the $\mathrm{Na}$ values. Dashes indicate that the element was not measured in that sample.

and 2 standard deviations, and 1 differed by 2 standard deviations. This pattern is close to the theoretical expectations $(11,4$, and 1 , respectively) and suggests that there were no severe fractionation problems in the measurement of the duplicate samples.

When samples from the identical interval were not ground together, the agreement is sometimes fairly good, as seen in Table 3 for Samples DSEA-32/32a (516F-89-5, 27-28 cm) and 35/35a (516F-89$5,34.0-34.5 \mathrm{~cm}$ ), but sometimes large differences in chemical abundances occur, as seen in Samples DSEA-26/26a (516F-89-5, 11-12.5 $\mathrm{cm}), 28 / 28 \mathrm{a}(516 \mathrm{~F}-89-5,18-20 \mathrm{~cm})$, and $29 / 29$ a (516F-89-5, 20-22 cm) for the elements $\mathrm{Cr}, \mathrm{Hf}, \mathrm{Ta}$, and $\mathrm{Th}$. These differences probably reflect changes in composition over a region of a millimeter or a few millimeters. Although the values given in the tables are precisely measured, ratios between elements should be taken with caution unless these elements were measured on the same pill or on pills made from powder ground together at the same time. There should be enough information given in the text and tables to make these distinctions.

\section{RESULTS AND CONCLUSIONS}

An iridium anomaly was detected in Sample $516 \mathrm{~F}$ $89-5,16-36.5 \mathrm{~cm}$. The maximum $\mathrm{Ir}$ abundance was 0.95 $\pm 0.18 \mathrm{ppb}$ in $516 \mathrm{~F}-89-5,33.0-33.5 \mathrm{~cm}$. In 23 measurements of the background in a region extending from 43 $\mathrm{m}$ above the anomaly to $48 \mathrm{~m}$ below, no Ir was detected, and the best value of the background was $0.017 \pm$ 0.031 or $<0.08 \mathrm{ppb}$. The Ir data are plotted in Figure 2 . From the Ir abundances within Sample 516F-89-5, 16$36.5 \mathrm{~cm}$ (Table 2), interpolations of the abundances in the unmeasured gaps, and a density of $2.2 \mathrm{~g}$ of dry sediment per $\mathrm{cm}^{3}$ wet sediment deduced from the water content (Schaftenaar et al., this volume), the total Ir in the lated to be $12 \mathrm{ng} / \mathrm{cm}^{2}$.

Thus this region of the South Atlantic does demonstrate a definite Ir anomaly associated with the Cretaceous/Tertiary boundary region. It is lower than the 30 $\mathrm{ng} / \mathrm{cm}^{2}$ found in DSDP Site 524 in the South Atlantic off the coast of Africa (Hsü et al., 1982; U. Krähenbühl, personal communication, 1981) but comparable to the $15 \mathrm{ng} / \mathrm{cm}^{2}$ in the first Cretaceous/Tertiary anomaly found at Gubbio, Italy (Alvarez et al., 1980).

The data in Tables 2 and 3 show that 18 of 25 elements peak strongly in part of the same region as the Ir (Sample 516F-89-5, 29-36.5 cm). Many of these peaks may be related to an enhancement of about $3 \mathrm{~g} / \mathrm{cm}^{2}$ of an aluminous noncalcareous mineral (probably clay). This amount of enhancement is about twice that of the Cretaceous/Tertiary boundary clay in Gubbio, Italy, and Stevns Klint, Denmark (Alvarez et al., 1980), and nearly comparable to that in DSDP Hole 465 (Michel et al., 1981; authors' unpublished data, 1982²). The abundances of $\mathrm{Cr}, \mathrm{Ti}$, and especially $\mathrm{U}$, are higher in the $\mathrm{Al}$ rich samples in the Ir region than in other clays in the section, and the abundance of $\mathrm{Mn}$ is lower.

Some deductions may be drawn concerning the origin of the Cretaceous/Tertiary boundary clay in Hole $516 \mathrm{~F}$ from the ratio of the $\mathrm{Ce} / \mathrm{Sm}$ abundances. The $\mathrm{Ce} / \mathrm{Sm}$ ratios for all of the samples studied in this work are plotted in Figure 3 along with the $\mathrm{Al}$ and some Sm abundances. As the detrital clay content in a marine section increases with respect to the authigenic and biogenic components, the $\mathrm{Ce} / \mathrm{Sm}$ ratio should increase because continental shales often have $\mathrm{Ce} / \mathrm{Sm}$ ratios of 10 or more, whereas seawater has a ratio of $\sim 1$. Thus the peaks in the $\mathrm{Ce} / \mathrm{Sm}$ ratio at the top and bottom of Figure 3, which also correspond roughly to peaks in the $\mathrm{Al}$

\footnotetext{
${ }^{2}$ Unpublished data on the Cretaceous/Tertiary region of Hole 465A. Recently available density data reduced by about one-third the estimates of Ir and clay content of this region as given by Michel and others, 1981.
} 
Table 3. Trace element abundances expressed in ppm (in ppb for Ir). ${ }^{\mathrm{a}}$

\begin{tabular}{|c|c|c|c|c|c|c|c|c|c|c|c|c|c|c|c|c|c|c|}
\hline $\begin{array}{l}\text { Sample } \\
\text { number }\end{array}$ & $\mathrm{Ba}$ & Co & $\mathrm{Cr}$ & $\mathrm{Ca}$ & $\mathrm{Hf}$ & $\operatorname{Ir}(\mathrm{ppb})^{\mathrm{b}}$ & $\mathrm{Mn}$ & $\mathrm{Ni}$ & $\mathrm{Rb}$ & sb & $\mathrm{Se}^{\mathrm{e}}$ & Se & St & $\mathrm{Ta}$ & Th & u & $\mathrm{v}$ & $\mathrm{Zn}^{\mathrm{c}}$ \\
\hline A-103 & $1130 \pm 21$ & $.81 \pm 0.12$ & \pm 0.7 & $52 \pm 0.07$ & $05 \pm 0.04$ & $<0.4(0.22 \pm 0.20)$ & $0 \pm 10$ & $32 \pm$ & $30.4 \pm 2.5$ & $25 \pm 0.05$ & $<0.37$ & $4.42 \pm 0$. & $84 \pm 269$ & $0.244 \pm 0.004$ & \pm 0.035 & 040 & $18 \pm 8$ & $.6 \pm$ \\
\hline o & 0 & & & & 20 & $10=077$ & & & $32.5 \pm 2.6$ & ses & & 30 & 312 & 0.176 & 034 & & & \\
\hline-105 & $840 \pm 17$ & $4.40 \pm 0.12$ & $3 \pm 0.6$ & $1 \pm 0.05$ & $0.544 \pm 0.006$ & 0 & $5 \pm 14$ & $15.4 \pm 3.2$ & $16.2 \pm 1.9$ & S.19 & 0.066 & $2.832 \pm 0.028$ & $1604 \pm 312$ & $0.117 \pm$ & $1.501 \pm 0.028$ & 0.925 & 35 & \\
\hline $\begin{array}{l}-106 \\
-107\end{array}$ & $\begin{array}{l}1074 \pm 20 \\
260 \pm 9\end{array}$ & $6.82 \pm 0.15$ & $4.9 \pm 0.6$ & $0.81 \pm 0.05$ & $0.526 \pm 0.031$ & $<0.4(-0.11 \pm 0.20)$ & $\begin{array}{l}532 \pm 11 \\
585+12\end{array}$ & $5.2 \pm 3.2$ & $\begin{array}{l}19.0 \pm 2.1 \\
29.8+2.6\end{array}$ & $\begin{array}{l}<0.15 \\
0.0400\end{array}$ & $<0.27$ & $2.351 \pm 0.024$ & $\begin{array}{l}1583 \pm 308 \\
1284+251\end{array}$ & $0.102 \pm 0.003$ & $1.327 \pm 0.027$ & $0.220 \pm 0.034$ & $<38$ & $19.7 \pm 2.3$ \\
\hline $\begin{array}{l}-107 \\
-22\end{array}$ & $\begin{array}{l}260 \pm 9 \\
464 \pm 14\end{array}$ & $\begin{array}{r}7.39 \pm 0.16 \\
1699+0.32\end{array}$ & $\begin{aligned} 9.1 & \pm 0.7 \\
47.2 & =1.1\end{aligned}$ & $\begin{array}{l}1.53 \pm 0.07 \\
6.21 \pm 0.15\end{array}$ & $\begin{array}{c}0.887 \pm 0.036 \\
4.63 \pm 0.08\end{array}$ & $\begin{array}{l}<0.5(-0.21 \pm 0.24) \\
<1.1(-0.29 \pm 0.53)^{\circ}\end{array}$ & $\begin{array}{l}585 \pm 12 \\
445 \pm 9\end{array}$ & $\begin{array}{c}17.5 \pm 3.9 \\
78 \pm 8\end{array}$ & $\begin{array}{c}29.8 \pm 2.6 \\
145 \pm 10\end{array}$ & $\begin{array}{l}0.34 \pm 0.06 \\
0.94 \pm 0.12\end{array}$ & $\begin{array}{l}<0.28 \\
<0.7\end{array}$ & $\begin{array}{l}3.660 \pm 0.037 \\
13.01 \pm 0.13\end{array}$ & $\begin{aligned} 1284 & \pm 251 \\
679 & \pm 84\end{aligned}$ & $\begin{array}{l}0.184 \pm 0.004 \\
0.976 \pm 0.010\end{array}$ & $\begin{array}{l}2.226 \pm 0.032 \\
10.55 \pm 0.11\end{array}$ & $\begin{array}{l}0.290 \pm 0.033 \\
1.047 \pm 0.020\end{array}$ & $\begin{array}{l}36 \pm 17 \\
97 \pm 18\end{array}$ & $\begin{array}{l}24.2 \pm 2.6 \\
104 \pm 5\end{array}$ \\
\hline${ }_{-23}^{-22}$ & $\begin{array}{l}464 \pm 14 \\
328 \pm 14\end{array}$ & $\begin{array}{l}16.99 \pm 0.32 \\
12.36 \pm 0.26\end{array}$ & 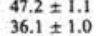 & $\begin{array}{l}6.21 \pm 0.15 \\
4.31 \pm 0.13\end{array}$ & $\begin{array}{l}4.63 \pm 0.088 \\
3.25 \pm 0.07\end{array}$ & $\begin{array}{l}<1.1(-0.29 \pm 0.033)^{*} \\
<0.8(0.02 \pm 0.39)^{\circ}\end{array}$ & $445 \pm 9$ & $\begin{array}{l}78 \pm 8 \\
41 \pm 7\end{array}$ & $\begin{array}{l}145 \pm 10 \\
111 \pm 8\end{array}$ & $\begin{array}{l}0.944 \pm 0.12 \\
0.47 \pm 0.09\end{array}$ & $\begin{array}{l}<0.7 \\
<0.9\end{array}$ & $\begin{array}{l}13.01 \pm 0.13 \\
14.64 \pm 0.15\end{array}$ & $679 \pm 84$ & $\begin{array}{l}0.976 \pm 0.010 \\
0.763 \pm 0.008\end{array}$ & $\begin{array}{r}10.55 \pm 0.11 \\
8.95 \pm 0.09\end{array}$ & $\begin{array}{l}1.047 \pm 0.020 \\
0.923 \pm 0.020\end{array}$ & $97 \pm 18$ & $\begin{aligned} 104 \pm 5 \\
80 \pm 4\end{aligned}$ \\
\hline-24 & $469 \pm 14$ & $25.0 \pm 0.4$ & $\begin{array}{l}55.3 \pm 1.2 \\
55.2\end{array}$ & $7.18 \pm 0.17$ & $4.80 \pm 0.08$ & $<1.2(-0.6 \pm 0.68)^{*}$ & $428 \pm 9$ & $73 \pm 8$ & $166 \pm 12$ & $0.72 \pm 0.12$ & $<0.5$ & $15.02 \pm 0.15$ & $432 \pm 65$ & $1.081 \pm 0.011$ & $11.63 \pm 0.12$ & $1.246 \pm 0.022$ & $111 \pm$ & $131+5$ \\
\hline-25 & 40 & 20.32 & 4 & 6.60 & $4.27 \pm 0.07$ & $\pm 0.5)^{\circ}$ & $0 \pm 8$ & 64 & is & & $<0.6$ & & & & it & & & $106 \pm 5$ \\
\hline .26 & 17 & & & & $0.604 \pm 0.033$ & & 14 & & & & $<0.34$ & & 594 & $0.144 \pm 0.004$ & $1.75 \pm 0.06$ & 0.234 & & \\
\hline$-26 a$ & & $4.37 \pm 0.12$ & $7.7 \pm$ & $1.22 \pm 0.06$ & $0.756 \pm 0.033$ & $<0.20(-0.14 \pm 0.10)$ & - & $21.0 \pm 3.9$ & $\begin{array}{l}26.7 \pm 2.5 \\
226 \pm 2 .\end{array}$ & $23+005$ & $<0$ & $3.631=0.036$ & $2+$ & $0.182 \pm 0.003$ & $1.969 \pm 0.031$ & & & \\
\hline $\begin{array}{l}-27 \\
-28\end{array}$ & $\begin{array}{l}246 \pm 10 \\
470+16\end{array}$ & $3.71 \pm 0.11$ & $\begin{array}{r}8.9 \pm 0.7 \\
14.55+3.0\end{array}$ & $1.01 \pm 0.06$ & $\begin{array}{l}0.683 \pm 0.032 \\
1.06 \pm 0.05\end{array}$ & $0.21 \pm 0.09^{\circ}$ & $\begin{array}{l}590 \pm 12 \\
559 \pm 11\end{array}$ & $\begin{array}{c}8.6 \pm 3.6 \\
-\end{array}$ & $22.6 \pm 2.9$ & $23 \pm 0.05$ & $\begin{array}{l}<0.26 \\
\leq 0.6\end{array}$ & $3.417 \pm 0.034$ & 682 & $0.152 \pm 0.003$ & $1.672 \pm 0.033$ & $0.24 \mathrm{C}$ & $16 \pm 7$ & $18.4 \pm$ \\
\hline $\begin{array}{l}-28 \\
-28 \mathrm{a}\end{array}$ & $470 \pm 16$ & $4.34 \div 0.12$ & $\begin{aligned} 14.5 & \pm 3.0 \\
8.8 & \pm 0.7\end{aligned}$ & $1.20 \pm 0.06$ & $\begin{aligned} 1.06 & \pm 0.05 \\
0.790 & \pm 0.034\end{aligned}$ & $<0.24(0.06 \pm 0.09)$ & $559 \pm 11$ & $8.5 \pm 3.8$ & $26.2 \pm 2.5$ & $17 \pm 0.05$ & $\begin{array}{l}<0.6 \\
<0.29\end{array}$ & $3.97 \pm \mathrm{c}$ & $714 \pm 92$ & $\begin{array}{l}0.264 \pm 0.005 \\
0.173 \pm 0.003\end{array}$ & $\begin{array}{r}2.55 \pm 0.09 \\
1.919 \pm 0.032\end{array}$ & $\begin{aligned} 0.403 & \pm 0.020 \\
- & \end{aligned}$ & $\begin{array}{l}31 \pm 9 \\
-\end{array}$ & $21.4 \pm 2$ \\
\hline $\begin{array}{l}-28 \mathrm{a} \\
.29\end{array}$ & $489 \pm 13$ & $4.34 \pm 0.12$ & $\begin{aligned} 8.8 & \pm 0.7 \\
14.3 & \pm 2.3\end{aligned}$ & $1.20 \pm 0.06$ & $\begin{array}{c}.790 \pm 0.034 \\
1.21 \pm 0.04\end{array}$ & $<0.24(0.00 \pm 0.09)$ & $536 \pm 11$ & $8.5 \pm 3.8$ & $26.2 \pm 2.5$ & $17 \pm 0.05$ & $\begin{array}{l}<0.39 \\
<0.34\end{array}$ & $3.97 \pm \mathrm{C}$ & $737 \pm 93$ & $\begin{array}{l}0.173 \pm 0.003 \\
0.280 \pm 0.004\end{array}$ & $\begin{array}{c}1.9919 \pm 0.032 \\
2.79 \pm 0.07\end{array}$ & $20 \pm 0$ & $42 \pm 10$ & $21.4 \pm 2$ \\
\hline$-29 \mathrm{a}$ & & $4.17 \pm 0.12$ & $9.2 \pm 0.7$ & $0.98 \pm 0.06$ & $0.752 \pm 0.034$ & $<0.33$ & & $8.6 \pm 3.8$ & $25.6 \pm 2.4$ & $21 \pm 0.04$ & $<0.4$ & $4.01 \pm$ & & & $1.758 \pm 0.031$ & & & $2 \pm$ \\
\hline .30 & $414 \pm 14$ & & $13.1 \pm 2.6$ & & $0.67 \pm 0.04$ & & $542 \pm 11$ & (1) & & & $<0$ & & $742 \pm 94$ & & & 17 & $33 \pm 11$ & $21.2 \%$ \\
\hline .31 & & $5.58 \pm 0.16$ & $14.5 \pm 0.8$ & $42 \pm$ & $0.89 \pm$ & $0.12)^{*}$ & & 16 & $33 \pm 4$ & 07 & $<0$ & $5.19 \pm 0.05$ & & 004 & & & & 22.8 \\
\hline-32 & 31 & $4.03 \pm 0.12$ & 10.3 .3 & 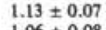 & $0.738 \pm$ & & 9 & 10 & 21.7 & & $<0$ & & 469 & $6 \pm 0.003$ & 2.010 & 013 & $<24$ & $15.6 \pm 2.5$ \\
\hline$-32 \mathrm{a}$ & & & 13 & & & $<0.24$ & $290+6$ & & & & & & & & & & -1 & \\
\hline $\begin{array}{l}-33 \\
-34\end{array}$ & $\begin{array}{l}543 \pm 14 \\
297 \pm \pm 11\end{array}$ & $\begin{array}{l}12.20 \pm 0.25 \\
9.48+0.21\end{array}$ & $\begin{array}{l}34.7 \pm 0.9 \\
27.8 \pm 0.9\end{array}$ & $\begin{array}{l}4.43 \pm 0.12 \\
2.78 \pm 0.09\end{array}$ & $\begin{array}{l}2.70 \pm 0.06 \\
1.80 \pm 0.05\end{array}$ & & $290 \pm 6$ & $\begin{array}{l}32 \pm 6 \\
30 \pm 5\end{array}$ & $\begin{array}{c}105 \pm 8 \\
65 \pm 5\end{array}$ & $\begin{array}{l}0.28 \pm 0.07 \\
0.12 \pm 0.05\end{array}$ & $<0.39$ & & $702 \pm 78$ & & & $\begin{aligned} 1.208 & \pm 0.020 \\
5.05 & \pm 0.05\end{aligned}$ & $\stackrel{37 \pm 11}{-}$ & $\begin{array}{l}73.4 \pm 3.7 \\
33.2+3.1\end{array}$ \\
\hline-1 & $\begin{array}{l}297 \pm \pm 11 \\
261 \pm 10\end{array}$ & $\begin{array}{l}9.48 \pm 0.21 \\
8.32 \pm 0.17\end{array}$ & $\begin{aligned} 27.8 & \pm 0.9 \\
24.9 & \pm 0.8\end{aligned}$ & $\begin{array}{l}2.78 \pm 0.09 \\
2.20 \pm 0.08\end{array}$ & $\begin{array}{l}1.80 \pm 0.05 \\
1.48 \pm 0.04\end{array}$ & & $385 \pm 8$ & $\begin{array}{l}30 \pm 5 \\
24 \pm 5\end{array}$ & $\begin{aligned} 65 \pm 5 \\
50.9 \pm 3.3\end{aligned}$ & $\begin{array}{l}0.12 \pm 0.05 \\
0.15 \pm 0.06\end{array}$ & $\begin{array}{l}0.35 \pm \\
<0.32\end{array}$ & $\begin{array}{l}7.24 \\
6.84\end{array}$ & $911 \pm 90$ & $\begin{array}{l}0.419 \pm 0.004 \\
0.339 \pm 0.004\end{array}$ & $\begin{array}{l}4.43 \\
4.43\end{array}$ & $\begin{array}{l}5.05 \pm 0.05 \\
1.98 \pm 0.05\end{array}$ & $28 \pm 8$ & $\begin{array}{l}33.2 \pm 3.1 \\
31.2 \pm 3.0\end{array}$ \\
\hline-114 & $224 \pm 9$ & $7.27 \pm$ & $\begin{array}{l}24.6 \pm \pm \\
24.9\end{array}$ & $2.04 \pm$ & $\begin{array}{l}1.37 \pm \\
1.37\end{array}$ & $<0$. & & 19 & 47.2 & 0.18 & $<0$ & & & & 4.43 & & $28 \pm 0$ & 24.1 \\
\hline-115 & & $14.52 \pm$ & M & $5.82=$ & $3.68 \pm$ & 0.95 & $\frac{3}{2}$ & & & & & & $\begin{array}{l}689 \pm 74 \\
689\end{array}$ & & & & $\begin{array}{l}20 \pm 8 \\
52 \pm 12\end{array}$ & \\
\hline-116 & 201 & $6.74 \pm$ & 18.0 & $1.60=$ & $0.933 \pm$ & 0.30 & $380 \pm 8$ & & 37.1 & $<0.16$ & $<$ & & $1072 \pm 103$ & & & & $28 \pm 11$ & 20.0 \\
\hline-30 & 410 & 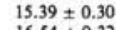 & 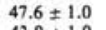 & 6.11 & 3.60 & & 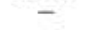 & & 12 & 0.29 & $<0<-<$ & & - & 010 & 7. & 030 & - & 75.8 \\
\hline$-35 \mathrm{a}$ & & & & & & $<$ & - & & & & $<$ & & & & & & & \\
\hline $\begin{array}{l}-36 \\
-37\end{array}$ & $\begin{array}{l}212 \\
254\end{array}$ & $\begin{array}{l}7.12 \pm \\
8.26 \pm\end{array}$ & $14.8 \pm$ & $\begin{array}{l}1.64 \pm 0.07 \\
1.97 \pm 0.07\end{array}$ & $0.992 \pm C$ & & 10 & ${ }_{28}^{14}$ & $\begin{array}{c}34.5 \pm 3.6 \\
44+4\end{array}$ & & $\begin{array}{l}0.41 \pm \\
<0.32\end{array}$ & & $\begin{array}{l}1092 \\
842\end{array}$ & & & & $\begin{array}{l}31 \pm 15 \\
33+7\end{array}$ & \\
\hline $\begin{array}{l}-37 \\
-38\end{array}$ & 25 & $\begin{array}{l}8.26 \pm \\
8.86 \pm\end{array}$ & $\begin{array}{l}15.8 \pm \\
14.8 \pm\end{array}$ & $\begin{array}{l}1.97 \pm \\
1.53 \pm\end{array}$ & $\begin{array}{r}1.26= \\
1.038=\end{array}$ & & & & 37.4 & & $\begin{array}{l}<0.32 \\
<0.5\end{array}$ & & & & & & $\begin{array}{l}33 \pm 7 \\
<21\end{array}$ & $\begin{array}{l}25.1 \pm 2.7 \\
27.4 \pm 2.7\end{array}$ \\
\hline $\begin{array}{l}-38 \\
-39\end{array}$ & & 9. & & & 1.04 & & 2 & 12 & 34.9 & & $<$ & & & 04 & & 14 & $26+1$ & $\begin{array}{l}21.4 \pm 2.7 \\
21.1+2.7\end{array}$ \\
\hline-40 & 26 & & 13 & 1.70 & $1.072 \pm$ & & \pm 12 & & & & & & $904 \pm$ & & & & $<48$ & $\begin{array}{l}2.7 \\
\end{array}$ \\
\hline- & & $14.52=$ & 15 & 2.05 & $1.26 \pm$ & & . & 27 & & & $<$ & & -7 & 04 & & & & 36 \\
\hline 8 & & & & & & & & & & & $<$ & & & & & & & \\
\hline & & & & & & & & & & & & & & & & & & \\
\hline $\begin{array}{l}-110 \\
-111\end{array}$ & 371 & 11 & 27. & $\begin{array}{l}3.1 \\
5.0\end{array}$ & 2. & & & ${ }_{48}^{22}$ & & & $<$ & & & & & & & \\
\hline-112 & $9 \pm 11$ & $\begin{array}{l}2 \pm 0.219 \\
0 \pm 0.19\end{array}$ & $21.3 \pm 0.8$ & $\begin{array}{l}3 \pm 0.09 \\
6\end{array}$ & $1.84 \pm 0.05$ & 8) & $f \pm 14$ & 15 & $.7 \pm$ & $34 \pm 0.07$ & $<0.5$ & .07 & 653 & \pm 0.005 & \pm 0.05 & $69 \pm 0.04$ & $1 \pm 14$ & $\begin{array}{l}38.5 \pm 3.3 \\
38.5\end{array}$ \\
\hline & & & & & & & & & & & & & & & & & & \\
\hline
\end{tabular}

a The indicated errors are the estimated precisions of measurement; i.e., essentially the standard deviations in the counting of gamma rays following activation by neutron irradiation. Because of uncertainties in interference corrections, all precisions are at least $1 \%$, altho
elements are smaller. The accuracies of the measurements are comparable to the precisions except when fux monitors were used for calibration. For corresponding DSDP sample numbers, see Table 1. Dashes indicate that the element was nor measured in the sample.

belements are smaller. The accuracies of the measurements are comparable to the precesions except when nux monitors were used for callbration. For corresponding DSDP sample numbers, see Table 1 . Dasthes indicate that the element was not measured in the sample.

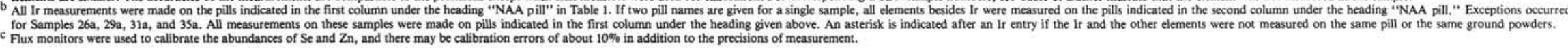




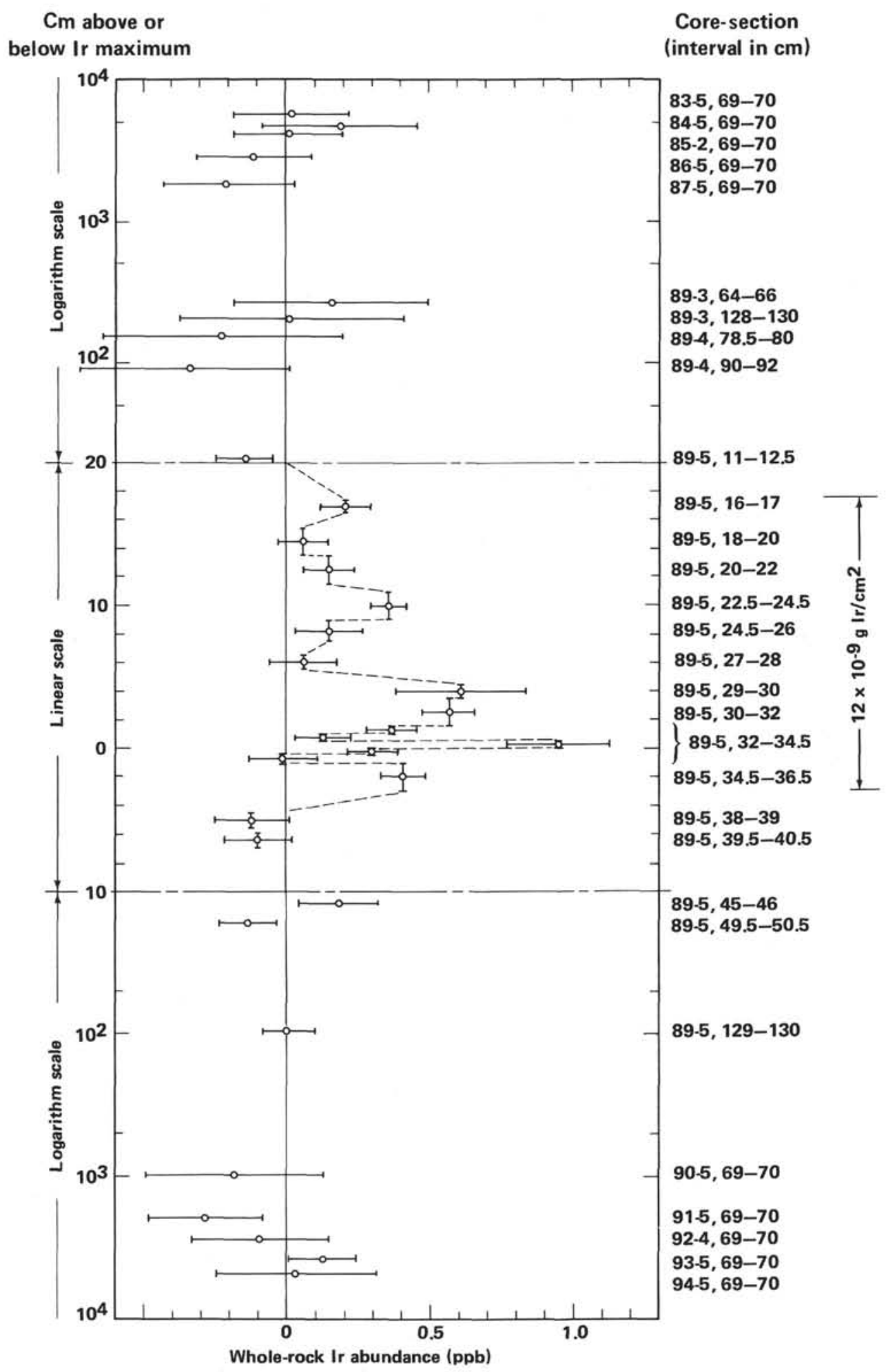

Figure 2. Iridium abundance profile in the Cretaceous/Tertiary boundary region of DSDP Hole 516F.

abundance, probably represent enhancements of detrital clay. The Ir region, on the other hand, contains three distinct $\mathrm{Al}$ abundance peaks with valleys in between but no corresponding oscillation in the $\mathrm{Ce} / \mathrm{Sm}$ ratio. In fact the $\mathrm{Ce} / \mathrm{Sm}$ ratio varies rather smoothly in the Ir region. It decreases about $30 \%$ over a span of $6 \mathrm{~cm}$ from a somewhat constant value of 12 in Cretaceous sediments just below the boundary to a value of 8.5 in Tertiary sediments just above. Thus the clay in the Ir region is different from the detrital clays above and below the boundary, and is thus probably not mostly detrital, i.e., it has a prominent authigenic component. 


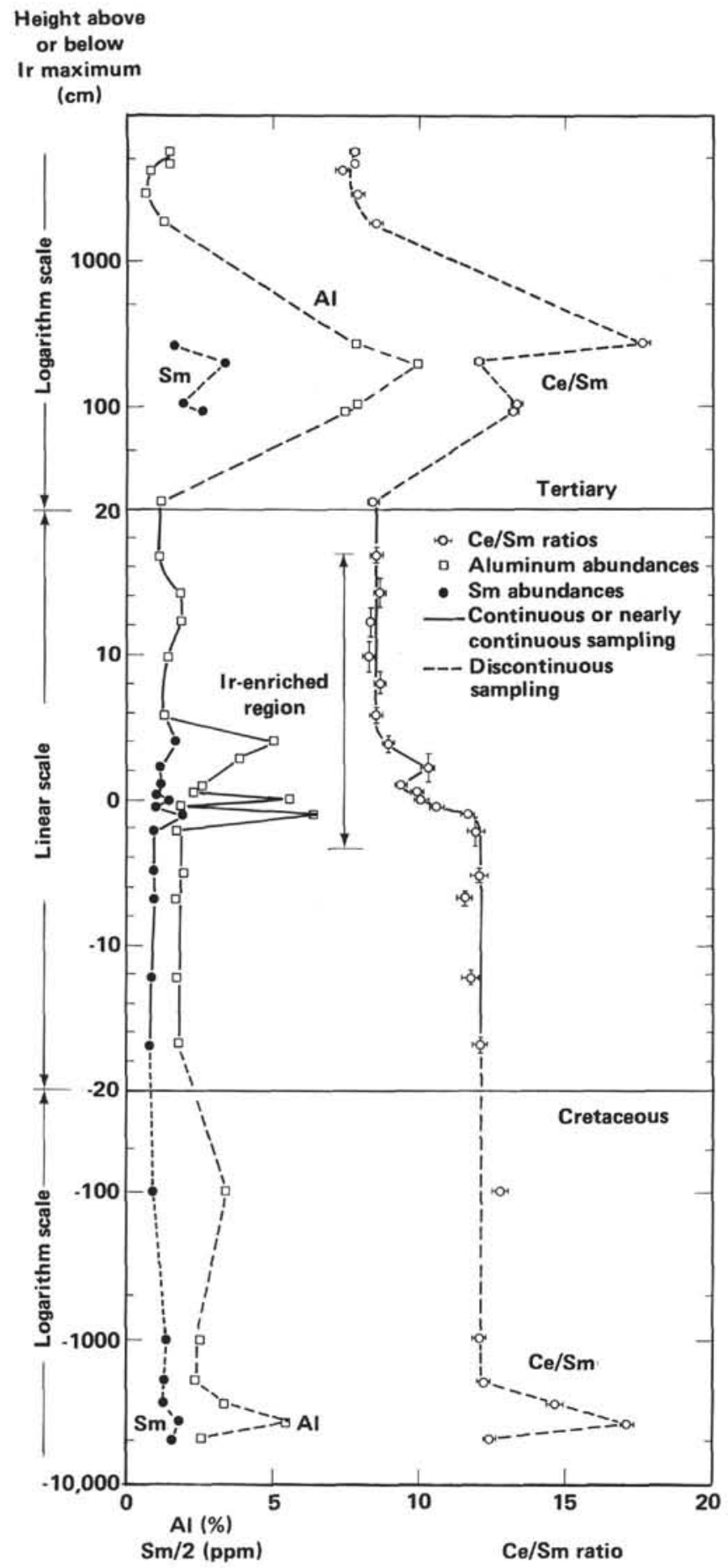

Figure 3. $\mathrm{Al}$ and $\mathrm{Sm}$ whole-rock abundance profiles and $\mathrm{Ce} / \mathrm{Sm}$ abundance ratios in the Cretaceous/Tertiary boundary region of DSDP Hole 516F.

Such a component would be expected if the Ir were the result of a large bolide impact $65 \mathrm{Ma}$, and if part of the materials deposited in the Ir-rich region were alteration products of glassy dust resulting from the impact.
We note that there is a very close correspondence between the iridium maximum and the "nannofossil" Cretaceous/Tertiary boundary at Site 516, and that the "foraminiferal" Cretaceous/Tertiary boundary is apparently several centimeters shallower in the core (Fig. 1). The lithology in the vicinity of the boundary does not indicate extensive upward or downward mixing by bioturbation or by the drilling process. The Cretaceous planktonic foraminifers Globotruncana spp. apparently co-occur with nannofossils from Zone NP1 in an interval several centimeters thick above the iridium anomaly. We note this apparent lag between the major nannofossil "turnover" and that of the planktonic foraminifers; we suggest that the Cretaceous/Tertiary boundary stratigraphy be examined on a similar fine-scale spacing of samples at other locations where the sequence appears relatively undisturbed.

\section{ACKNOWLEDGMENTS}

Neutron irradiations were kindly provided by Tek Lim, supervisor of the small U. C. Berkeley research reactor, and his staff. This work was done with support from the Department of Energy under Contract DE-AC03-76SF00098, the California Space Institute under Award CS24-81 and the NASA Ames Research Center under Contract A$71683 \mathrm{~B}$. The DSDP samples were supplied through the assistance of the National Science Foundation. This is Contribution No. 5196 of Woods Hole Oceanographic Institution.

\section{REFERENCES}

Alvarez, L. W., Alvarez, W., Asaro, F., and Michel H. V., 1980. Extraterrestrial cause for the Cretaceous-Tertiary extinction. Science, 308:1095-1108.

Alvarez, W., Asaro, F., Alvarez, L. W., and Michel, H. V., in press. Current Status of the Impact Theory for the Terminal Cretaceous Extinction: Boulder, CO (Geol. Soc. Am. Spec. Publ.)

Alvarez, W., Asaro, F., Michel, H. V., and Alvarez, L. W., 1982. Iridium anomaly approximately synchronous with terminal Eocene extinctions. Science, 216:886-888.

Asaro, F., Michel, H. V., and Burger, R., 1981. Chemical source groups in Ecuadorian obsidian. Lawrence Berkeley Lab. Preprint $L B L-13247$.

Hsü, K. J., He, Q., McKenzie, J. A., Weissert, H., Perch-Nielsen, K., Oberhansli, H., Kelts, K., LaBrecque, J., Tauxe, L., Krähenbühl, U., Percival, S. F., Jr., Wright, R., Poore, R. Z., Gombos, A. M., Pisciotto, K., Karpoff, A. M., Petersen, N., Tucker, P., Carman, M. F., Jr., and Schreiber, E., 1982. Mass mortality and its environmental and evolutionary consequences. Science, 216:249-256.

Michel, H. V., Asaro, F., Alvarez, W., and Alvarez, L. W., 1981. Distribution of iridium and other elements near the Cretaceous/Tertiary boundary in Hole 465A: preliminary results. In Thiede, J., Vallier, T. L., et al., Init. Repts. DSDP, 62: Washington (U. S. Govt. Printing Office), 847-849.

Perlman, I., and Asaro, F., 1969. Pottery analysis by neutron activation. Archaeometry, 11:21-52.

1971. Pottery analysis by neutron activation. In Brill, R. H. (Ed.), Science and Archaeology: Cambridge, MA (MIT Press), pp. 182-195.

Yellin, J., Perlman, I., Asaro, F., Michel, H. V., and Mosier, D. F., 1978. Comparison of neutron activation analysis from the Lawrence Berkeley Laboratory and the Hebrew University. Archaeometry, 20:95-100.

Date of Initial Receipt: March 16, 1982 